\title{
Treatment of paraneoplastic neurological syndromes with antineuronal antibodies (Anti-Hu, Anti-Yo) with a combination of immunoglobulins, cyclophosphamide, and methylprednisolone
}

\author{
F Keime-Guibert, F Graus, A Fleury, R René, J Honnorat, P Broet, J-Y Delattre
}

Department of Neurology, Hôpital de la Salpêtrière, Paris, France

F Keime-Guibert

A Fleury

J-Y Delattre

Unité INSERM 495,

Hôpital de la

Salpêtrière, Paris,

France

J-Y Delattre

Service of Neurology

CS "Principes

Espana" Hospitalet del

Llobregat, Spain

R René

Service of Neurology, Hospital Clinic i provincial, Barcelona, Spain

F Graus

Service of Neurology B and Inserm U 433, Hôpital Neurologique,

Lyon, France

J Honnorat

Unité INSERM 472. Hôpital P, Brousse, Villejuif Cedex, France P Broet

Correspondence to: Dr Jean-Yves Delattre, Service de Neurologie, Hôpital de la Salpêtrière, 47 Boulevard de l'Hôpital, 75651 Paris Cedex 13,

France

Received 22 March 1999 and in revised form

25 August 1999

Accepted 3 September 1999

\begin{abstract}
Objectives-To evaluate the effect of a combination of immunoglobulins (IVIg), cyclophosphamide (CTX), and methylprednisolone (MP) on the clinical course of patients with paraneoplastic neurological syndrome (PNS) and antineuronal antibodies (Abs).

Methods-Seventeen patients with paraneoplastic encephalomyelitis/sensory neuropathy (PEM/SN) with anti-Hu Abs $(n=10)$ or cerebellar degeneration (PCD) with anti-Yo Abs $(n=7)$ received one to nine cycles (mean 3.5) of a combination of IVIg ( $0.5 \mathrm{~g} / \mathrm{kg} /$ day from days 1 to 5$)$, CTX $(600 \mathrm{mg} / \mathrm{m} 2$ at day 1$)$ and MP (1g/day from day 1 to 3 ). The Rankin scale (RS) was used to evaluate the response. A positive response was considered as either improvement or stabilisation in patients who were still ambulatory $(R S \leqslant 3)$ at the onset of treatment, whereas only improvement, and not stabilisation, was considered a therapeutic benefit in bedridden patients (RS $\geqslant 4)$.
\end{abstract}

Results-Tolerance was good and no patient experienced grade 3/4 toxicity (World Health Organisation). Sixteen patients were evaluable for response. Of the seven patients with $R S \geqslant 4$, none improved. Of the nine patients with $R S \leqslant 3$, none improved but three (two $\mathrm{SN}$ and one PCD) stabilised for 4,35 , and 16 months.

Conclusions-This study suggests that vigorous immunosuppressive treatment is not useful in severely disabled PNS patients with antineuronal Abs. In a minority of patients (mainly with $\mathrm{SN}$ ) who are not severely disabled at the onset of treatment, a transient stabilisation is possible and deserves further evaluation.

(F Neurol Neurosurg Psychiatry 2000;68:479-482)

Keywords: paraneoplastic neurological syndromes; antineuronal $\mathrm{Ab}$; immunosuppresive treatment

The presence of high titres of antineuronal antibodies (Abs) directed against antigens present in both neurons and tumour cells suggest that some paraneoplastic neurological syndromes such as paraneoplastic cerebellar degeneration (PCD) with anti-Yo Ab (also known as APCA) or paraneoplastic ence- phalomyelitis/sensory neuronopathy (PEM/ $\mathrm{SN}$ ) with anti-Hu Ab (also known as ANNA-1) are of autoimmune origin. Unfortunately, with a few exceptions, ${ }^{1-6}$ immunosuppressive treatment such as plasmapheresis, corticosteroids, intravenous immunoglobulin (IVIg), or cyclophosphamide $(\mathrm{CTX})^{78}$ have been disappointing when used alone. However, there is evidence that a combination of IVIg+CTX+methylprednisolone (MP) could be a more efficient approach in various autoimmune diseases, particularly in diseases with an intrathecal synthesis of Ig. ${ }^{9}$ As intrathecal synthesis of autoantibodies is a frequent finding in PCD with anti-Yo $\mathrm{Ab}$ and in $\mathrm{PEM} / \mathrm{SN}$ with anti-Hu Ab, ${ }^{10}{ }^{11}$ the effect of a multimodality immunosuppressive treatment associating CTX, MP, and IV Ig was evaluated in a series of 17 patients.

Patients and methods

PATIENT CHARACTERISTICS

Seventeen patients (11 women and six men) aged 50 to 72 years (median=65), with PCD with anti-Yo $\mathrm{Ab}(\mathrm{n}=7)$ or $\mathrm{PEM} / \mathrm{SN}$ with anti-Hu $\mathrm{Ab} \quad(\mathrm{n}=10)$ were treated with IVIg+CTX+MP between 1994 and 1996 (table).

Of 10 patients with anti-Hu Ab, four had CNS involvement (limbic encephalitis, brainstem encephalitis, cerebellar degeneration) and six had a sensory neuronopathy without CNS involvement. Three of these six patients also had signs of dysautonomia.

The seven patients with PCD and anti-Yo $\mathrm{Ab}$ had an isolated pancerebellar syndrome.

The median delay between the development of paraneoplastic symptoms and the onset of treatment was 10 months for anti-Hu+ patients (range 3 to 20 months), and 3 months for antiYo+ patients (range 0.5 to 5 months). In all cases, the neurological disorder had progressed within the 2 months preceding the therapeutic trial.

NATURE AND TREATMENT OF THE PRIMARY TUMOUR

A tumour was found in all patients as indicated in the table. In the anti-Hu+ patients, the neurological syndrome always preceded the tumour diagnosis (median time 7.5 months; range 3-41). In the anti-Yo+ patients, the neurological syndrome occurred after the tumour diagnosis in three patients (median 
Table 1 Clinical features of patients treated with IVIg, CTX, MP

\begin{tabular}{|c|c|c|c|c|c|c|c|c|c|c|c|c|c|}
\hline & Sex/age & Tumour $\left({ }^{\star}\right)$ & $\begin{array}{l}\text { Ab } \\
\text { type }\end{array}$ & $\begin{array}{l}\text { Delay } \\
\text { PNS/ } \\
\text { Tumour } \\
(\text { mo })(t)\end{array}$ & $\begin{array}{l}\text { Clinical } \\
\text { picture }(\ddagger)\end{array}$ & $\begin{array}{l}\text { Delay } \\
\text { PNS/ } \\
\text { Tx (\$) }\end{array}$ & $\begin{array}{l}\text { Tumour } \\
\text { treatment } \\
\text { during Tx (\) }\end{array}$ & $\begin{array}{l}\text { RS at } \\
\text { onset } \\
\left({ }^{\star \star}\right)\end{array}$ & $\begin{array}{l}\text { Number } \\
\text { of cycles }\end{array}$ & $\begin{array}{l}\text { Effect of } T x \\
\text { on PNS } \\
(+t)\end{array}$ & $\begin{array}{l}\text { Toxicity } \\
\text { of Tx }\end{array}$ & $\begin{array}{l}\text { Survival } \\
\text { after Tx } \\
\text { (mo) }\end{array}$ & Cause of death \\
\hline 1 & $\mathrm{~F} / 67$ & SCLC+Breast & $\mathrm{Hu}$ & $41+$ & SN & 6 & None & 3 & 9 & Stable & No & 35 & Onco \\
\hline 2 & $\mathrm{M} / 62$ & SCLC & $\mathrm{Hu}$ & $4+$ & AN-SN & 6 & RT & 3 & 5 & Stable & No & 4 & Onco \\
\hline 3 & $\mathrm{M} / 62$ & Neuroendoc rectal & $\mathrm{Hu}$ & $9+$ & SN-LE & 12 & None & 1 & 2 & PD & No & 4 & Onco \\
\hline 4 & $\mathrm{~F} / 57$ & SCLC & $\mathrm{Hu}$ & $10+$ & BE-PCD & 10 & $\begin{array}{l}\text { Etoposide } \\
\text { Cisplatine } \\
\text { Epirubicine }\end{array}$ & 3 & 4 & PD & No & 10 & Onco \\
\hline 5 & $\mathrm{M} / 60$ & SCLC & $\mathrm{Hu}$ & $10+$ & $\mathrm{SN}$ & 9 & $\begin{array}{l}\text { Carboplatine } \\
\text { Etoposide }\end{array}$ & 3 & 3 & PD & No & 4 & Neuro \\
\hline 6 & $\mathrm{~F} / 71$ & SCLC & $\mathrm{Hu}$ & $5+$ & AN-SN & 12 & None & 2 & 2 & No eval & No & 3 & Onco \\
\hline 7 & $\mathrm{~F} / 67$ & SCLC metastasis & $\mathrm{Hu}$ & $3+$ & AN-SN & 3 & $\begin{array}{l}\text { Etoposide } \\
\text { Carboplatine }\end{array}$ & 4 & 3 & Stable & No & 4 & Onco \\
\hline 8 & $\mathrm{M} / 52$ & SCLC & $\mathrm{Hu}$ & $6+$ & SN-LE-BE & 6 & None & 1 & 2 & PD & No & $35+$ & Alive \\
\hline 9 & $\mathrm{M} / 72$ & Urothelial carcinoma & $\mathrm{Hu}$ & $6+$ & SN-LE & 20 & None & 4 & 5 & Stable & Allergy & 26 & Onco \\
\hline 10 & $\mathrm{M} / 50$ & SCLC & $\mathrm{Hu}$ & $12+$ & $\mathrm{SN}$ & 19 & None & 4 & 5 & Stable & No & 12 & Onco \\
\hline 11 & $\mathrm{~F} / 67$ & Ovarian & Yo & $43-$ & PCD & 0.5 & None & 3 & 1 & PD & No & 5 & Neuro \\
\hline 12 & $\mathrm{~F} / 70$ & Ovarian & Yo & $5+$ & PCD & 5 & $\begin{array}{l}\text { Carboplatine } \\
\text { Cyclophos }\end{array}$ & 3 & 3 & PD & No & 17 & $\begin{array}{l}\text { Lost to follow } \\
\text { up }\end{array}$ \\
\hline 13 & $\mathrm{~F} / 52$ & Breast & Yo & $6-$ & PCD & 4 & None & 3 & 1 & Stable & No & 16 & Onco \\
\hline 14 & $\mathrm{~F} / 65$ & Ovarian & Yo & $2+$ & PCD & 3 & $\begin{array}{l}\text { Cis-/ } \\
\text { Carboplatine } \\
\text { Adriamycine } \\
\text { Cyclophos. }\end{array}$ & 4 & 2 & PD & No & 2 & Neuro \\
\hline 15 & $\mathrm{~F} / 67$ & Ovarian & Yo & $24-$ & PCD & 1 & None & 4 & 3 & Stable & No & 2 & Suicide? \\
\hline 16 & $\mathrm{~F} / 54$ & Breast & Yo & $3+$ & PCD & 5 & $\begin{array}{l}\text { Fluorouracil } \\
\text { Epirubicine } \\
\text { Cyclophos } \\
\text { RT }\end{array}$ & 4 & 7 & Stable & No & $38+$ & Alive \\
\hline 17 & $\mathrm{~F} / 70$ & $\begin{array}{l}\text { Unknown origin } \\
\text { metastasis }\end{array}$ & Yo & $5+$ & PCD & 5 & $\begin{array}{l}\text { Cisplatine } \\
\text { Etoposide }\end{array}$ & 4 & $2 ?$ & PD & No & 2 & Not known \\
\hline
\end{tabular}

(*) Tumour: SCLC= small cell lung cancer.

$(\dagger)$ Delay between the occurence of the paraneoplastic neurological syndrome (PNS) and discovery of the tumour.

( + the PNS precedes the tumour; - the PNS follows the tumour).

( ) $\mathrm{AN}=$ autonomic neuropathy; $\mathrm{SN}=$ sensory neuronopathy; $\mathrm{LE}=$ limbic encephalitis; $\mathrm{PCD}=$ paraneoplastic cerebellar degeneration; $\mathrm{BE}=$ brainstem encephalitis.

( () Delay between the occurence of PNS and the treatment by Ig-corticoids-cyclophosphamide.

(ब) $\mathrm{Tx}=$ treatment by IgG-corticosteroids-cyclophosphamide; RT= radiotherapy; Cyclophos=cyclophosphamide.

$(\star \star)$ Rankin scale at onset.

(†十) $\mathrm{PD}=$ progressive disease.

time 24 months; range 6-43) and before the diagnosis of the tumour in four patients (median time 4 months; range 2-5).

In patients with anti-Hu $\mathrm{Ab}$, the tumour histology was small cell lung cancer (eight patients), neuroendocrine tumour of rectum (one), urothelial carcinoma (one). One patient with small cell lung cancer also had a breast adenocarcinoma. In patients with anti-Yo $\mathrm{Ab}$, the primary tumours were breast (two), ovarian (four), and metastasis of an unknown primary site (one).

Eight patients had treatment of the tumour (chemotherapy and/or radiotherapy) during immunosuppressive therapy. Among the nine others, six had received antineoplastic treatment before immunonsuppressive therapy, and three after.

IMMUNOSUPPRESSIVE TREATMENT

All seventeen patients received a combination of IVIg, $0.5 \mathrm{~g} / \mathrm{kg} /$ day for five days (day 1 to day 5), intravenous MP, $1 \mathrm{~g} /$ day for 3 days (day 1 to day 3), and intravenous CTX, $600 \mathrm{mg} / \mathrm{m}^{2}$ for 1 day (day 4). If the patient was improved or stable after the first cycle, the treatment was repeated three times at 3 week intervals. After the third treatment, if the patient was improved or stable, he received a "maintenance" treatment of IV Ig $(0.5 \mathrm{~g} / \mathrm{kg})$, intravenous MP (1g), and intravenous CTX $(600 \mathrm{mg} / \mathrm{m})$ delivered in 1 day, monthly for 6 months.

Neurological impairment was evaluated by a modified Rankin scale ${ }^{12}(0=$ asymptomatic patient; $1=$ non-disabling symptoms which do not interfere with lifestyle; $2=$ minor disabling symptoms, which lead to some restriction of lifestyle but do not prevent totally independent existence; $3=$ symptoms significantly interfere with lifestyle or prevent totally independent existence; $4=$ moderately severe disabling symptoms which clearly prevent independent existence with total support for basic daily activities; $5=$ severe disabling, totally dependent requiring constant attention day and night; $6=$ death from neurological symptoms). $\mathrm{Pa}-$ tients were considered improved or deteriorated if their Rankin score (RS) changed by at least 1 point. Stabilisation was defined as no change of RS after three courses of therapy.

Survival was determined from onset of treatment to death. The median survival time was defined as the shortest survival time for which the value of the Kaplan-Meier estimator of the survival function was $<0.5 .^{13}$

The detection of anti-Hu and anti-Yo Abs was done by indirect immunoperoxidase on frozen sections of normal human brain cortex or cerebellum, and confirmed by western blot analysis. Serum and CSF anti-Hu and anti-Yo titres were defined as the reciprocal value of the highest dilution that demonstrated neuronal nuclear or Purkinje cell staining. Antibody titrations were done before treatment in serum and in some patients in CSF. When possible, titrations were repeated after treatment.

\section{Results}

Patients received one to nine cycles of immunosuppressive therapy (mean 3.5 ). Tolerance was good and no patient experienced grade $3 / 4$ 
toxicity (World Health Organisation). Only one patient (9) had a slight reaction characterised by feeling of discomfort with shivering and reversible agitation at the end of the second cycle, which did not recur during the subsequent cycles.

One patient (6) was not evaluable for therapeutic response; he developed symptomatic brain metastasis, and it was not possible to distinguish the respective responsibilities of the paraneoplastic syndrome and of the metastasis in his neurological deterioration.

Of the nine evaluable anti-Hu+ patients, none improved, five (56\%) stabilised, and four $(44 \%)$ deteriorated. Among the six patients who presented with an $\mathrm{RS} \leqslant 3$ at the beginning of the treatment four $(3,4,5,8)$ deteriorated and the two others, including one who also received antitumour treatment, were stabilised (4, 35 months). The three anti-Hu+ patients who had an RS $>3$ at the beginning of the treatment $(7,9,10)$ were stabilised.

All anti-Yo+ patients were evaluable for therapeutic response. Three of them presented with $\mathrm{RS} \leqslant 3$ at the onset. One stabilised during 16 months (13) and two worsened (11, 12). The four patients who had RS $>3$ at presentation $(14,15,16,17)$ deteriorated.

Post-treatment autoantibody titres in serum samples were available in only five anti-Hu+ patients and two anti-Yo+ patients. In all but one patient, the titres dropped substantially. Mean pretreatment and post-treatment titres were respectively 53200 (SD 46 639) and 15 400 (SD 15 868) in anti-Hu+patients. In the two anti-Yo+ patients, decreased titres after the treatment were found in one patient (32000 to 8000 ) whereas the titre did not change after three courses of treatment in the other patient. Autoantibody titres in CSF could be evaluated twice in only one anti-Hu+ patient (7), in whom it decreased after three courses from 500 to 100. Unfortunately, this patient with a sensory neuropathy had no intrathecal synthesis of the anti- $\mathrm{Hu} \mathrm{Ab}$ at the onset according to Schuller's formula. ${ }^{14} 15$

Thus, of the 16 evaluable patients, none improved and only three (18.8\%), two patients with SN and one with PCD experienced "useful stabilisation", defined as stable disease when RS remained $\leqslant 3$. Median survival for all the patients (after the onset of the treatment) was 5 months (range 2-38+ months). It was 6 (range 3-35+) and 5 (range $2-38+$ ) months for the anti-Hu+ and the anti-Yo+ patients, respectively.

\section{Discussion}

The response of the paraneoplastic neurological syndromes to immunosuppressors or antitumour treatment is greatly influenced by the underlying neuropathology. When the neuronal cell bodies are spared such as in LambertEaton myasthenic syndrome, paraneoplastic vasculitic neuropathy, and some patients with paraneoplastic opsoclonus-myoclonus, recovery has been reported after immunotherapy and anticancer therapy. ${ }^{16-18}$ Unfortunately, the hallmark of PEM/SN with anti-Hu $\mathrm{Ab}$ and of $\mathrm{PCD}$ with anti-Yo $\mathrm{Ab}$ is a neuronal loss.
In this setting, a therapeutic benefit can be considered as either improvement, which implies that some kind of functional alterations may precede neuronal death, or at least stabilisation in patients who are still ambulatory $(\mathrm{RS} \leqslant 3)$. In bedridden patients $(\mathrm{RS} \geqslant 4)$ only improvement is relevant and stabilisation cannot be considered a therapeutic benefit because it is useless in terms of quality of life and may occur spontaneously. ${ }^{19}$

Using these criteria for response, the purpose of this study was to evaluate the effect of a vigorous immunosuppressive treatment combining high doses of steroids, CTX, and IVIg in PNS with anti-Hu or anti-Yo Ab. This regimen was selected because each agent has been occasionally claimed to be useful in $\mathrm{PNS}^{4}{ }^{20-22}$ raising the hope that their combination could be additive or synergistic. Furthermore, such combinations have been successful in other autoimmune diseases, including diseases with intrathecal synthesis of IgG, a feature that is also found in many patients with paraneoplastic neurological syndromes and antineuronal Ab. ${ }^{11}$

Despite a good tolerance, our results are clearly disappointing in the patients who were severely disabled at the onset of treatment $(\mathrm{RS} \geqslant 4)$ as none of them improved suggesting that immunosuppressors are not useful in this subgroup, whatever the type of paraneoplastic neurological syndromes (PCD or PEM/SN). The most likely hypothesis to explain this finding is that the treatment was started too late at a stage when neuronal loss was already massive and irreversible. Lack of efficacy of immunosupressors in disabled patients has been previously found by our group and others ${ }^{78}$ in 30 patients identified in the literature and in our database. However, Oh et al recently reported on two severely disabled patients with PEM/SN with anti-Hu $\mathrm{Ab}$ who improved after immunosuppressors alone (steroids in one and steroids+IVIg+azathioprine in the other $)^{6}$ but some caution is necessary to interpret this finding because two cases of spontaneous improvement have also been described in the anti-Hu syndrome. ${ }^{23}$ In fact, improvement after treatment with immunosuppressors in severely disabled patients seems so exceptional (if it does exist) that we think that systematic prescription of very expensive agents (IVIg) or potentially harmful agents that may interfere with chemotherapy of the underlying tumour (CTX) is not indicated in patients with $\mathrm{RS} \geqslant 4$. Nevertheless, a trial of corticosteroids may be worthwhile in this setting according to the literature.

Analysis of the patients who were still ambulatory $(\mathrm{RS} \leqslant 3)$ at the onset of treatment is more complex. In these patients, none of them improved but two out of six with an anti-Hu syndrome (both had $\mathrm{SN}$ ) and one out of three PCD with anti-Yo Ab stabilised for 4, 35, and 16 months, respectively. The "useful" stabilisation experienced by these patients could result from two possibilities. Firstly, the immunosuppressor treatment may have had a real, albeit modest effect that could not be ascribed only to the tumour treatment which was not concomitantly administered in two of these three patients. If this is the case, our data suggest that 
in the anti-Hu syndrome, immunosuppressors would mainly benefit patients with involvement of the peripheral nervous system. In a previous study we also found that a few patients with $S N$ (two of six with $\mathrm{RS} \leqslant 3$ ) apparently benefited from IVIg. ${ }^{8}$ It is of interest to note that five out of the six patients with an anti-Hu syndrome who have been reported to improve after various immunosuppressors (and antitumour treatment in five cases) had an exclusive involvement of the peripheral nervous system with sensory neuropathy as the main finding,,$^{1-3568}$ leading to the suggestion that the lack of a blood-brain or blood-nerve barrier at the level of the dorsal root ganglia could facilitate the entry and action of immunosuppressors in patients with $\mathrm{SN}$. The second possibility is that our three patients had an indolent form of the disease ${ }^{24}$ or had a spontaneous stabilisation, an issue that we identified in previous studies. ${ }^{8}$ Even if we took care to avoid this bias by selecting only patients who were deteriorating neurologically when immunosuppressive therapy was started, we cannot rule out a coincidental association between treatment onset and spontaneous stabilisation of a disease the natural history of which may be heterogeneous. The fact that serum titres of antineuronal $\mathrm{Ab}$ dropped substantially in six out of the seven patients in whom we obtained serum samples before and after treatment, including three patients who received immunosuppressors alone, could favour the hypothesis of a real beneficial effect of immunosuppressors. Nevertheless, such findings have been made previously after plasmapheresis or IVIg without a clinical counterpart ${ }^{7825}$ and we were not able, as others, ${ }^{12526}$ to find a correlation between serum titres of $\mathrm{Ab}$ and neurological course. For example, patient 1 who experienced "useful" stabilisation had a 10 -fold fall in serum titre after treatment but patient 4 also had a striking drop of serum $\mathrm{Ab}$ (16-fold) despite progressive neurological deterioration. We cannot comment on the effect of this therapeutic regimen on intrathecal synthesis of the autoantibodies as the only patient in this series who had repeated CSF examination had pure $\mathrm{SN}$ and had no intrathecal synthesis of the anti-Hu $\mathrm{Ab}$, in agreement with our previous findings. ${ }^{11}$

Only a prospective randomised study could definitely answer to the question of a possible benefit of immunosuppressive therapy. Unfortunately, as previously stated, it is highly unlikely that a double blind randomised trial can ever be designed in these disorders due to their extremely low frequency. At least, analysis of series coming from the same institutions provide more information than simple case reports. Despite its methodological limitations, this trial indicates that combined treatment with IVIg, CTX, and MP in patients with $\mathrm{PEM} / \mathrm{SN}$ or PCD with antineuronal $\mathrm{Ab}$ is well tolerated but does not seem to be useful in debilitated patients $(R S \geqslant 4)$. The question remains open of a transient stabilisation in this regimen in a minority of the patients (mainly with $\mathrm{SN}$ ) who are still ambulatory at the onset of treatment $(\mathrm{RS} \leqslant 3)$. To better delineate the respective roles of immunosuppressors and antitumour treatment in these diseases, we are currently screening our database to see if the addition of immunosuppressive therapy to antitumour treatment modifies the neurological and oncological course compared with antitumour treatment alone, particularly in patients with $\mathrm{SN}$.

This work was supported by the "Association pour la Recherche sur les Tumeurs Cérébrales". We thank Drs M Uchuya, C Kouhatra, PJ Souquet, B Guillon, and JH Ruel for their help in hatra, PJ Souquet, B Guillon, and JH Ruel for their help in patient follow up and collecting data, and Dr B Benyahia, MC
Nadaud, L Tömpe, B Duprey, and I Lysson for their technical help.

1 Younger DS, Dalmau J, Inghirami G, et al. Anti-Huassociated peripheral nerve and muscle microvasculitis. Neurology 1994;44:181-3.

2 Verschuren J, Twijnstra A, De Baets M, et al. Hu antigens and anti-Hu antibodies in a patient with myxoid chondrosarcoma. Neurology 1994;44:1551-2.

3 Twijnistra A, Verschuuren J, Byrne TN, et al. Prolonged or remitting anti-Hu associated paraneoplastic sensory neuronremithy and encephalomyelitis. Neurology 1995;45(suppl opathy and

4 Stark E, Wurster U, Patzold U, et al. Immunological and clinical response to immunosuppressive treatment in paraneoplastic cerebellar degeneration. Arch Neurol 1995;52:814-18.

5 Gabriel CM, Gregson NA, Hughes RAC. Sensory neuropathy and anti-Hu antibodies in a patient with seminoma. Eur F Neurol 1996;3:471-4.

$6 \mathrm{Oh}$ SJ, Dropcho EJ, Claussen GC. Anti-Hu associated paraneoplastic sensory neuronopathy responding to early
aggressive immunotherapy: report of two cases and review of literature. Muscle Nerve 1997;20:1576-82.

7 Graus F, Vega F, Delattre JY, et al. Plasmapheresis and antineoplastic treatment in CNS paraneplastic syndromes with antineuronal autoantibodies. Neurology 1992;42:536-40.

8 Uchuya M, Graus F, Vega F, et al. Intravenous immunoglobulin treatment in paraneoplastic neurological syndromes with antineuronal antibodies. F Neurol Neurosurg Psychiatry 1996; 60:388-92.

9 Hommes O R, Lamers KJB, Van Geel W. Intrathecal IgG synthesis and IgG index after intensive and chronic immunosuppressive treatment of multiple sclerosis. Ann NY Acad Sci 1984;436:410-17.

10 Furneaux HM, Reich L, Posner JB. Autoantibody synthesis in the central nervous system of patients with paraneoplastic syndromes. Neurology 1990;40:1085-91.

11 Vega F, Graus F, Chen QM, et al. Intrathecal synthesis of the anti-Hu antibody in patients with paraneoplastic encephalomyelitis or sensory neuronopathy: clinical-immunologic correlation. Neurology 1994;44:2145-7.

12 Van Doorn PA, Brand A, Strengers PWF, et al. High-dose intravenous immunoglobulin treatment in chronic inflammatory demyelinating neuropathy: a double blind, placebo controlled, crossover study. Neurology 1990;40:209-12.

13 Kaplan EL, Meier P. Non-parametric estimation from incomplete observations. Fournal of the American Statistical Association 1958;53:457-81.

14 Schuller E, Benabdallah S, Sagar HJ, et al. IgG synthesis within the central nervous system. Comparison of three formulas. Arch Neurol 1987;44:600-4.

15 Schuller E. A new strategy for the study of intrathecal immunity. In: Marrosu MG, Cianchetti C, Tavolato B, eds. Trends in neuroimmunology. New-York: Plenum, 1990:3-12.

16 Newsom-Davis J, Murray NMF. Plasma exchange and immunosuppressive drug treatment in the Lambert-Eaton myasthenic syndrome. Neurology 1984;34:480-5.

17 Chalk CH, Murray NMF, Newsom-Davis J, et al. Response of the Lambert-Eaton myasthenic syndrome to treatment of associated small-cell lung carcinoma. Neurology 1990;40: $1552-6$

18 Hammer MS, Larsen MB, Stack CV. Outcome of children with opsoclonus-myoclonus regardless of etiology. Pediatr Neurol 1995;13:21-4.

19 Dalmau J,Graus F, Rosenblum MK, et al. Anti-Hu associated paraneoplastic encephalomyelitis/sensory neuronopathy: a paraneoplastic encephalomyelitis/sensory neuronopa
clinical study of 71 patients. Medicine 1992;71:59-72.

20 Moll JWB, Henzen-Logmans SC, Van der Meche FGA. Early diagnosis and intravenous immune globulin therapy in paraPsychiatry 1993;56:112

21 Counsell CE, McLeod M, Grant R. Reversal of subacute paraneoplastic cerebellar syndrome with intravenous immunoglobulin. Neurology 1994;44:1184-5.

22 Glantz MJ, Biran H, Myers ME, et al. The radiographic diagnosis and treatment of paraneoplastic central nervous system disease. Cancer 1994;73:168-75.

23 Byrne T, Mason WP, Posner JB, et al. Spontaneous neurological improvement in anti-Hu associated encephalomyelitis. $\mathcal{F}$ Neurol Neurosurg Psychiatry 1997;62:276-8.

24 Graus F, Bonaventura I, Uchuya M, et al. Indolent anti-Hu-associated paraneoplastic sensory neuropathy. Neurology 1994;44:2258-61.

25 Ben David Y, Warner E, Levitan M, et al. Autoimmune paraneoplastic cerebellar degeneration in ovarian carcinoma patients treated with plasmapheresis and immunoglobulin. patients treated with plasm

26 Yagi Y, Inuzuka T, Takada K, et al. Changes in anti-Hu antibody titers in the long term course in paraneoplastic sensory neuropathy. Eur Neurol 1997;37:122-3. 